\title{
Keyseg: adaptive segmentation for spontaneous electroencephalography map series into spatially defined microstates of musicians' brain
}

\author{
Indra K. Wardani ${ }^{1}$, Phakkharawat Sittiprapaporn², Djohan ${ }^{3}$, Fortunata Tyasrinestu ${ }^{4}$, Prayoon \\ Suyajai ${ }^{5}$ \\ 1,3,4 Indonesia Institute of the Arts, Yogyakarta, Indonesia \\ ${ }^{2}$ Neuropsychological Research Laboratory, Department of Anti-Aging and Regenerative Science, School of Anti-Aging \\ and Regenerative Medicine, Mae Fah Luang University, Bangkok, Thailand \\ ${ }^{2}$ Brain Research Unit for Anti-Aging and Regenerative Medicine, School of Anti-Aging and Regenerative Medicine, Mae \\ Fah Luang University, Bangkok, Thailand \\ ${ }^{5}$ Department of Psychology, Faculty of Humanities, Mahachulalongkornrajavidyalaya University, Phra Nakhon Si \\ Ayutthaya, Thailand
}

\section{Article Info}

Article history:

Received Aug 4, 2020

Revised Mar 5, 2021

Accepted May 29, 2021

\section{Keywords:}

Brain

Electroencephalography

Gendhing Lancaran

Karawitan

Microstate

\begin{abstract}
Music is being studied related to either its impact on the psychological interaction or cognitive process behind it. These examinations bring out music's coordination to numerous disciplines including neuroscience. A few past examinations exhibited the contrast among musicians and non-musicians regarding brain structure and brain activity. The current investigation exhibited the diverse brain activation while musicians tuned in to music with regards to their musical experiences utilizing microstate classes method analysis. The investigation intended to determine electroencephalography microstate changes in Karawitan musicians' brain while tuning in to Gendhing Lancaran. Applying the electroencephalography microstate investigation of Karawitan musicians, the occurrence parameters was computed for four microstate classes (A, B, C, and D). Microstate properties were compared among subjects and correlated to Gendhing Lancaran perception. The present results revealed that Karawitan musicians' brain were characterized by microstate classes with the increased prominence of classes A, B, and D, but decreased prominence of classes $\mathrm{C}$ while tuning in to Gendhing Lancaran. Our finding is the first study to identify the typical microstate characteristics of the Karawitan musician's brains while tuning in to Gendhing Lancaran by using the microstate segmentaion method.
\end{abstract}

This is an open access article under the CC BY-SA license.

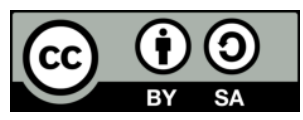

\section{Corresponding Author:}

Phakkharawat Sittiprapaporn

Neuropsychological Research Laboratory, Department of Anti-Aging and Regenerative Science

School of Anti-Aging and Regenerative Medicine, Mae Fah Luang University

Bangkok, Thailand

Email:wichian.sit@mfu.ac.th

\section{INTRODUCTION}

As neuroscience is known as a discipline to explain the mechanism that lies behind the perception and reception of music, it is utilized as a discipline to comprehend the impact of specific musical activities on the brain structurally and functionally focusing on its cognizance interaction for quite a long time. A few past examinations had been done to investigate the impact produced by music activity on psychological and cognitive function. One of the past investigations revealed that tuning in to music passively as a background 
of certain activity has been demonstrated to improve psychological execution and cognitive function [1]. Additionally, some previous investigations revealed how musical training was beneficial to alter brain function and improved psychological execution and cognitive function outside music. For example, certain treatment of music by mean of tempo and mode was able to excite arousal and mood to enhance psychological and cognitive capacities in spatial tasks. By applying the visuospatial tasks to both symphonic orchestral musicians and non-musicians further provided proof on how complexity in musical training done by symphonic orchestral musicians can influence the activation of Broca's area which enhanced their performance [1]-[3]. Regarding to the structure of the human brain, Gaser and Schlaug effectively exhibited how the human brain structure was diversed by comparing among professional musicians, amateur musicians, and non-musicians. This previous investigation expected a relative contricution of long-term musical practice to be done by both amateur and professional musicians as the fundamental cause [4]. In addition, some previous investigations showed that the musical experience turned into a significant thought as prior conditioning to separate an individual by comparing musicians and non-musicians [1], [5]. The fundamental argumentation of this assumption was depended on the notion that the involvement of longlasting musical training and certain skills acquirement experienced by individual empower them to have an alternate cognitive process. Musicians experience through complex rehearsal processes including physical and mental activities to interpret musical notation as a piece of visual information and alter it as a motoric reaction, to recall the musical expression, to do musical improvisation, and to recognize a note without any referential note [5]. All those complexities in music rehearsal were considered as brain stimuli to train its function and enhance its performance as well as develop its perception [1], [5].

Electroencephalography (EEG) is an appropriate technique for the investigation of global states and because of its high time resolution, it is additionally completely appropriate for the investigation of the briefer states (microstates) embedded therein [6]. Global states can be depicted utilizing proportions of dimensional complexity. These are indicators of the number of independent brain processes dynamic in the brain [7] and have for instance been applied to describe gross states, for example, sleep stages [8]. Global states vary between normal healthy controls and depressed [9] and schizophrenic patients [10] and have been proposed as proportions of brain development [11]. EEG frequency spectra and source localization examinations have been utilized in a few previous investigations. The most consistent discoveries were a diminished alpha and increased delta and theta frequency band activity. The EEG can be taken at as electric potential distributions on the head surface ('scalp maps') at each moment in time. The EEG microstate analysis looks for scalp maps that show quasi-stable electric potential distributions over prolonged periods of time, called 'microstates' [6], [12]. In utilizing the EEG's very high time resolution, the microstate examination reveals microstate durations typically in the range of 60-120 milliseconds [6], [12]. Diverse scalp potential distributions more likely than not been created by various spatial distributions of neuronal electric activity in the brain. In this way, it is sensible to expect that distinctive microstates embody various sorts of data processing. EEG microstates have been referred to as putative 'atoms of thought and emotion' [13], [14]. A few classes of microstates have been distinguished [15], [16]. In spontaneous resting-state EEG, normally four classes of microstates are found [17] that are typically marked A, B, C, and D. The microstates of the various classes can be described utilizing parameters, for example, their number of occurrances, how long they cover and their mean term. These parameters commonly contrast between various states and task conditions. Changes of the microstate parameters have been accounted for various microstate classes in normal states of consciousness including non-REM sleep-all classes [18], modalities of thinking- all classes [19], personality characteristics-classes B and C [20], self-related/unrelated thoughts, mental arithmeticclasses B, C and D [21], altered states of consciousness, for example, contemplation-class B [22], all classes [23], and hypnosis-classes A, C, and D [24], yet additionally disorders like schizophrenia-all classes [25]-[30], euthymic bipolar disorder-class A [31], and frontoparietal dementia-class C [32]. Accordingly, the microstate analysis appears to be particularly encouraging for recognizing contrasts in attention-related, mentation-related, and reality-testing-related processing.

The idea of symbolization with regards to brain recordings is profoundly interwoven with the proposition of microstates in [33]. Lehmann found in ERPs and spontaneous EEG time arrangement, that the scalp potential maps display quasi-stable behavior enduring from tens to hundreds of milliseconds [6], [33] with sharp transition between them. Lehmann apportioned these time arrangements and assigned discrete symbols. As indicated in [28], the EEG representation regarding microstate classes is likewise a productive data reduction method. Since this discrete symbolization time-series could reflect activations from various neural populations it is reasonable to expect that each one of these microstates participates in various functions or cognitive tasks [6], [34]. Additionally, it has been resolved that only four microstates (comparative across all subjects) with original topographies dominate ([12] and [35]) the spontaneous EEG recording in healthy adults while alerting [17], [36]-[38]. It is extremely fascinating to note that these microstates are very common in many studies that investigate and utilize this microstate framwork. While the microstates viewed as the "atoms of thoughts"; essential constructing blocks of cognition and neural

Keyseg: adaptive segmentation for spontaneous electroencephalography map series ... (Indra K. Wardani) 
information processing in the human brain, the investigation of their scale-free dynamics demonstrate that they may be the basis for the rapid reorganization and adaptation of the functional networks of the brain [39]. It is normal and generally appreciated for scientists to assess cognition tasks through the microstates framework by prototyping the scalp potential maps and investigate their spatial properties. The microstates framework is a successful strategy to evaluate the human brain electric field in the time domain [28]. Referring to one previous investigation of musical preference and cognitive style showed that a tendency of individual who have certain cognitive styles as a base of their personality traits and how musical genres turned into a distinctive variable. Rather than inspected brain activity contrasts among musicians and nonmusicians, the current investigation planned to depict the brain electrical activities of Karawitan musicians' brains while tuning in to traditional Javanese music, Gendhing Lancaran, to better understand the Karawitan musicians' brains electric changes related to the complex music perception and cognition by utilizing EEG measures of microstates.

\section{RESEARCH METHOD}

\subsection{Participants}

EEG data of 20 healthy Karawitan musicians were accessible for the current investigation. All Karawitan muscians were healthy right-handed adults with normal hearing and no known neurological disorders. Participants comprised of 11 males and 9 females. They aged 23-29 (mean age: 28.25 years, $\mathrm{SD}=1.41$ ). All participants involved in the current investigation actively learned practical music lessons of Gendhing Lancaran for consecutive three years. All participants had been clarified and had affirmed the applied procedures of the current investigation. The investigation was completed as per the ethical standard set forth in the Helsinki Declaration. The approval of the Graduate School of the Indonesia Institute of the Arts, Yogyakarta, Indonesia, on experiment and written consent from each participant were obtained.

\subsection{Stimuli}

Gendhing Lancaran, traditional music of Java, was used as timuli in the current investigation. The sounds were presented binaurally via earphones at $85 \mathrm{~dB}$ SPL for 20 minutes per session. Two sessions were recorded from each participant with a 5 minutes break between recording sessions. Participants were told to focus on the stimuli presented via earphones.

\subsection{Electroencephalographic recording}

The recordings of all participants comprised the standard fourteen-channels EEG. The electrode placement depended on the international 10-20-system: AF3, F3, F7, FC5, T7, P7, O1, O2, P8, 8, FC6, F8, F4, and AF4, plus ground. All electrodes were referenced against left and right mastoids. EEG data was recorded with an Epoc Plus (EMOTIV, San Francisco, USA), via wireless neuroheadset. EEGs were acquired as continuous signals. EEGs were amplified with a gain of 30,000 and filtered with a bandpass of 0.1-100 Hz. The recordings were filtered and deliberately examined for eye movement and muscle artifacts. Epochs with voltage variation surpassing $\pm 100 \mu \mathrm{V}$ at any EEG channel were dismissed from further investigation. All brain responses were recalculated offline against average reference for further analysis. EEG signal recording was time-locked to the onset of stimuli.

\subsection{Data analysis}

\subsubsection{Data pre-processing}

From the controlled task-free resting EEG data, artifacts-free epochs were chosen after minimizing ocular artifacts by regression analysis in the time domain utilizing an automatic artifact identification method [40] and after subsequent visual inspection for remain artifacts. The following pre-processing steps were applied to the data considering the microstate analysis. The available 14-channel artifact-free EEG data were segmented into 2-s epochs. The available data were then re-referenced to the average reference and FFT-filtered between $2-20 \mathrm{~Hz}$, as generally applied for microstate analyses [16]. The collected data were converted, produced .edf file, and then converted into .csv file by the TestBench program. These data recordings were compatible with Microsoft Excel where they were compatible with spatial analysis. In order to measure the electric strength (hilliness) of a brain electric field map independently from its spatial configuration, the global field power (GFP) peak measure was applied in this investigation. The GFP peak measure was equivalent to the spatial standard deviation of all voltage estimations of one spontaneous EEG map. In the event that a potential map was very hilly, the GFP peak measure would be higher than if the potential map was relatively flat. The GFP as characterized above was independent of reference [41], [42]. For each participant, mean GFP peak amplitudes over participants were computed and all mean spontaneous EEG map series were carefully inspected for artifacts. The grand mean GFP peak amplitudes over 
participants were then computed. For all mean spontaneous EEG map series, the locations of the centroid of each spontaneous EEG map series were computed according to Wackermann's study [38]. Centroids were the points of gravity of the positive and the negative areas of an average reference-referred map. For each of these centroid location points, the location coordinates were determined on the left-right axis and on the anterior-posterior axis. All subsequent analysis steps (segmentation of the data into microstates and statistical analysis) were based on these extracted spatial descriptors of the maps.

\subsubsection{Microstate analysis}

The EEG microstate analysis technique has been reviewed by Michel and Koenig [16]. Following the classical procedure for microstate computation, in an initial step, the global field power (GFP) [43], curve was computed in each 2-s epoch. All potential maps at times of maximal GFP, named GFP-peaks, were chosen across all epochs per participant. The GFP-peak maps address potential maps at times of optimal signal-to-noise ratio and around GFP-peaks map topographies remain stable while they change in GFPtroughs [15]. Accordingly, in a subsequent advance, just these GFP-peak maps were then taken into a modified k-means clustering algorithm [44] that utilizes global map dissimilarity (GMD) [43] as clustering criterion and that produced for each participant four individual model maps, named microstate classes, that best clarified the variance in the information. The utilization of a cross validation criterion has recently shown that four model maps comprise an optimal solution [28]. In a third step, the individual model maps of all participants were utilized to compute to mean group model maps utilizing a permutation algorithm that maximizes the common variance over participants [28]. The sets of four group model maps were then used to compute a set of four grand mean model maps or classes using the same procedure. In order to remain comparable to previous publications, these grand mean classes were then related in a fourth step to the four normative microstate classes published by Koenig et al. [44] and were labeled accordingly as microstate classes A, B, C and D. In a final step, all GFP peak maps were attributed to one of the four grand mean classes. A 'microstate' is defined by consecutive GFP-peak maps of the same class. The start of each microstate is defined by the midpoint between the last GFP-peak map of the preceding microstate and the first GFP-peak map of the current microstate, and analogous for the end of each microstate. Since the start of the first microstate and the end of the last microstate of each 2-s epoch is unknown, the first and the last microstates were omitted for the computation of the typical microstate parameters: occurrence, coverage over time and duration. The 'occurrence' is the mean number of occurrences of a certain class per second across all epochs. The 'coverage' is the mean percentage of time covered by a certain microstate class across all occurrences of that class per second across all epochs. The 'duration' is the mean duration of a certain microstate class of all its occurrences per second across all epochs. The microstate analysis was computed using keyseg, developed at the KEY Institute for Brain-Mind research, Zurich [28], [45].

\subsection{Statistical analysis}

While Karawitan musicians tuning in to Gendhing Lancaran, electric activities of the Karawitan musicians' brains were constantly recorded. The statistical analysis was performed on GFP peak amplitudes of 14 electrode sites within the time range of EEG waveform $(0-55,000 \mathrm{msec})$. The statistical significance of electrical activities of each microstate class was tested with a two-way sample $t$-test. Post-hoc unpaired $t$-tests for an unequal variance were utilized to untangle the main effects.

\section{RESULTS AND DISCUSSION}

Considering the progressive widespread neuronal activation comprising of a motor and psychological execution or cognitive function of the musicians' brain, we anticipated wide-ranging contrasts of microstate EEG measures in Karawitan musicians' brains. We initially noticed extensive changes in all EEG measures of Karawitan musicians' brains. Moreover, we discovered correlation patterns between psychological execution or cognitive performance and EEG measures. As anticipated, the EEG microstate analysis demonstrated changes in the parameters of all four microstate classes in Karawitan musicians' brains while tuning in to Gendhing Lancaran, a traditional Javanese music. These outcomes suggest that the neuronal activation of Karawitan musicians' brains is reflected in information processing in the Karawitan musicians' brains. In addition, the main findings were an increase in at least one parameter for microstate classes A, B, and D, and a decrease for classes C. The fact that all four microstate classes may reflect the complex neuronal activation of Karawitan musicians' brains while tuning in to Gendhing Lancaran, a traditional Javanese music. The functional significance of the four microstate classes and of their interaction is not yet completely established but has recently been evoked to several coming studies. In the accompanying sections, our investigation outcomes are discussed in the light of what is known about the functional significance of the diverse microstate classes. 
The four grand mean microstate classes see Figure 1 extracted from the EEG. Microstate segmentation of electrical activities in the Karawitan musicians' brain while tuning in to Gendhing Lancaran showed four grand mean model maps of the four microstate classes labeled A-D as shown in Figure 2. The two-way sample t-test comparing grand mean microstates across participants were statistically significant $(0.85 \pm 0.36 \mu \mathrm{V} ; t(19)=10.67, p<0.0001)$. However, a repeated measures ANOVA conducted on the grand mean microstate classes of each microstate class did not yield the main effects of groups $\left(\mathrm{F}_{3,16}=0.81, p=0.50\right.$; Table 1). The post-hoc paired $t$-tests for the microstate parameters revealed those classes for which the parameters significantly contrasted between each grand mean microstate class as shown in Table 1.

Class A

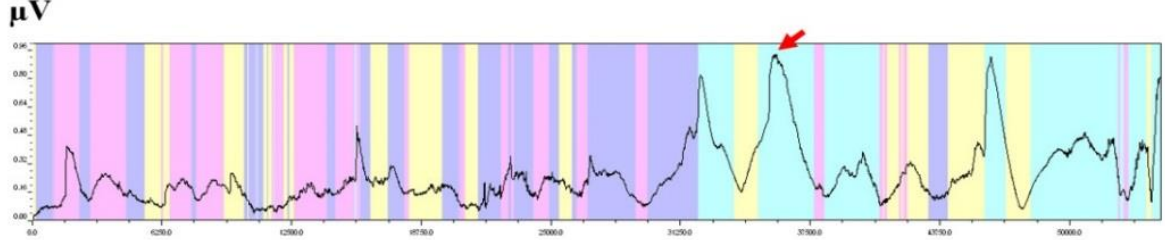

Class B

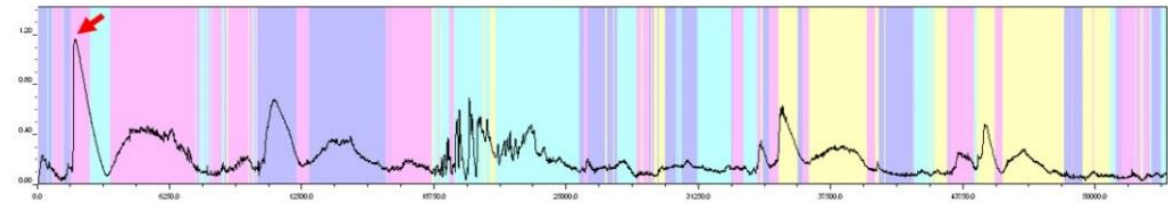

Class C

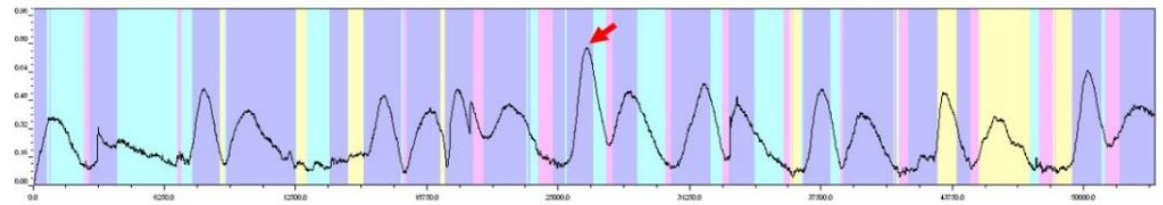

Class D

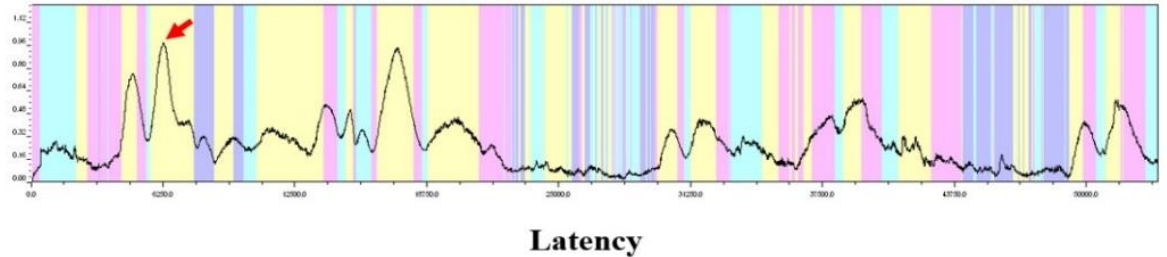

Figure 1. The classical methodology for microstate computation

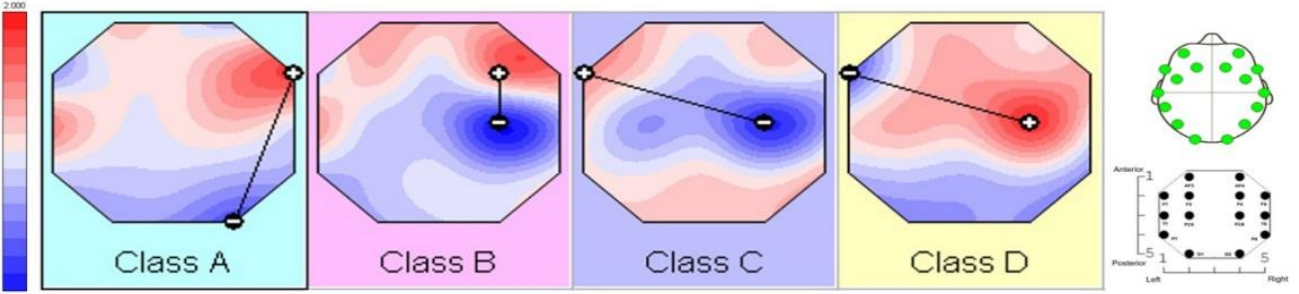

Figure 2. Representation of a typical 4-class EEG microstate topography sequence

The global field power (GFP) curve was computed and all potential maps at times of maximal GFP (red arrow) were chosen across all epochs per participantThese GFP-peak maps were then taken into a modified k-means clustering algorithm that utilized global map dissimilarity as a clustering criterion and that created for each participant four individual model maps, named microstate classes, that best clarified the variance in the EEG data. The utilization of a cross validation criterion has recently shown that four model maps comprise an optimal solution and arrangement [28].

As depicted in Figure 2, these four microstates (classes) depict particular topographies: Class A enhanced right-frontal and suppressed right-occipital activities; Class B enhanced right frontal and suppressed right centro-temporal activities; Class $\mathrm{C}$ enhanced left frontal and suppressed right centro- 
temporal activities; and Class $\mathrm{D}$ enhanced right centro-temporal and suppressed left frontal activites, respectively. Class $\mathrm{D}$ had most noteworthy electrical activities $(1.13 \pm 0.23 \mu \mathrm{V}, t(1)=7.06 ; p=0.089)$ contrasted with other classes (e.g., Class A: $0.99 \pm 0.27 \mu \mathrm{V}, t(2)=6.48 ; p=0.02$, Class B: $0.84 \pm 0.33 \mu \mathrm{V}, t(4)=5.66$; $p=0.004$, and Class C: $0.75 \pm 0.40 \mu \mathrm{V}, t(9)=5.92 ; p=0.0002)$, respectively.

Opposing polarity is represented by the arbitrarily designated colors momentary potential distribution of centroids positive and negative of each grand mean microstate classes across participants. The grand mean model maps of the four microstate classes. Equipotential area maps are shown. Semi-schematic electrode array in inset. Top right: Schematic of the electrode array on the scalp; head seen from above, left ear left. All electrodes were positioned according to the Epoc Plus (EMOTIV, San Francisco, USA), via wireless neuroheadset. The isopotential contour maps show the (arbitrarily assigned) areas of oppositye polarity in red and blue (normalized voltagevalues). A-D: microstate class labels. Bottom right: Tick marks at the coordinate axes indicate electrode numbers, in the anterior-posterior direction (vertical axis) and the leftright direction (horizontal axis). Centroids of the positive and negative map area computed for spatial feature extraction. The configuration of the map was described by the anterior-posterior and left-right coordinates of the centroid extreme positive and negative area.

Table 1. Microstate parameters per microstate class: means, standard deviations and $p$-value of paired post

\begin{tabular}{ccccc}
\hline \multicolumn{5}{c}{ hoc t-tests } \\
\hline Mean & Class A & Class B & Class C & Class D \\
SD & 0.99 & 0.84 & 0.75 & 1.13 \\
$p$-value & 0.27 & 0.33 & 0.40 & 0.23 \\
\hline
\end{tabular}

Table 1 shows grand mean microstate classes elicited in this investigation. The paired $t$-test comparing each grand mean microstate class was not statistically significant (Class A $(0.99 \pm 0.27)$ vs. Class B $(0.84 \pm 0.33), t(6)=0.69, p=0.52, \mathrm{~d}=-0.15$; Class A $(0.99 \pm 0.27)$ vs. Class $\mathrm{C}(0.75 \pm 0.40), t(11)=0.97, p=0.35$, $\mathrm{d}=-0.24$; Class A $(0.99 \pm 0.27)$ vs. Class D (1.13 \pm 0.23$), t(3)=0.58, p=0.60, \mathrm{~d}=-0.13$; Class B $(0.84 \pm 0.33)$ vs. Class C $(0.75 \pm 0.40), t(13)=0.42, p=0.68, \mathrm{~d}=-0.09$; Class B $(0.84 \pm 0.33)$ vs. Class D $(1.13 \pm 0.23), t(5)=1.11$, $p=0.32, \mathrm{~d}=0.29$; Class $\mathrm{C}(0.75 \pm 0.40)$ vs. Class $\mathrm{D}(1.13 \pm 0.23), t(10)=1.25, p=0.24, \mathrm{~d}=0.38)$. The directional changes of the microstate parameters of each grand mean microstate class for all comparisons were also illustrated in Table 2.

Table 2 illustrates the directional changes of the microstate parameters of each grand mean microstate class for all comparisons

\begin{tabular}{ccccc}
\hline & Class A & Class B & Class C & Class D \\
\hline Directional changes & $\downarrow$ & $\downarrow$ & $\rightarrow$ & $\leftarrow$ \\
\hline
\end{tabular}

In Karawitan musicians' brains, we noticed a statistically significant increased occurrence of classes A and B. Class A occurrence was increased in Karawitan musicians' brains, and correlations connected class A occurrence to motor function. We additionally found a pattern towards the increased class B occurrance, but we found no distinction in occurrence contrasted to class A in our Karawitan musicians' brains. In any case, we noticed non-statistical significantly increased occurrence of class D. Moreover, class B alterations have been accounted for in various normal, altered, and diseased states of consciousness. While class B diminishes altogether in all parameters were accounted for in light hypnosis [24], the duration was diminished in schizophrenic patients [29], [32], the coverage was increased in schizophrenic patients [25], occurrence and coverage were increased in believers in paranormal phenomena when contrasted with skeptics [20] and duration were increased in exceptionally experienced meditators during profound reflection [22], we discovered our Karawitan musicians' brains increased in class B occurrence which was the opposite direction of these previous concerns. Our reported findings appear to be more encouraging for the interpretation of increased class B occurrence corresponded with psychological execution or cognitive performance. A few investigations meant to relate microstate classes A and B with modality-specific, visual, or verbal/phonological processing [37], [43], [46], [47]. Recent findings recommend that class A prominence was increased when alpha inhibition was increased over left posterior language-related areas, and class B prominence was increased when alpha inhibition was increased over right posterior visuospatial-related areas [43]. Subsequently, our noticed increases in class A and B microstate prominence in Karawitan musicians' brains may mirror an increased inhibition and thus decrease both verbal and visual functioning in Karawitan musicians' brains.

Keyseg: adaptive segmentation for spontaneous electroencephalography map series ... (Indra K. Wardani) 
Class $\mathrm{C}$ microstates have been related with action in two significant centers of the default mode network (DMN) including the anterior cingulate cortex (ACC) [48] and the posterior cingulate cortex (PCC) [49]. A prior investigation revealed class $\mathrm{C}$ microstate correlations with positive blood oxygen level dependent (BOLD) signals in the ACC, the bilateral frontal gyri, and the right insula [50], regions belonging to the resting state network [51]. These areas cover with the saliency network and were connected to emotional interoceptive-autonomic processing. Class $\mathrm{C}$ processing consequently conceivably is engaged with the coordination of interoceptive data with emotional salience to shape a subjective own body representation [37]. In the current investigation, Karawitan musicians showed the diminished occurrence of class C microstate. Considering this finding, one may guess that in Karawitan musicians the progressive motor function decline and enthusiastic or emotional salience attribution is mostly identified with microstate class $\mathrm{C}$ processing. This could be due to the expanding attention-requesting parts of the coordination and involuntary movement over the span of Gendhing Lancaran tuning in. Curiously, class C microstates also showed diminished prominence, potentially due to the expanded internal focus on substantial insights and attention to bodily perceptions [24]. This is in accordance with Pipinis et al. [52] who reported a negative correlation of class C microstate coverage with experienced substantial mindfulness. As referenced above, Milz et al. [43] revealed that the recurrence band-wise source localization contrasts between the microstate classes are predominantly characterized by the alpha activity; likewise, class D showed the most elevated alpha activity. Most EEG studies revealed an increase in alpha activity in music perception, which appears to be an impression of being a characteristic marker of music tuning in. Thus, our finding of the increased in class D prominence is in line accordance with these observations.

In general, it is conceivable to accept that the interplay between the microstate classes is critical for the constitution of various normal, abnormal, and unhealthy conditions of consciousness. In a microstate study on hypnosis [24], light hypnosis showed a diminished occurrence and coverage of class $\mathrm{C}$ contrasted with resting while in profound hypnosis occurance and coverage of class A was expanded and length and coverage of class D diminished. Hypnosis has been described as engaged attention, enhanced somatic and enthusiastic control, and absence of self-conscious [53]. It has additionally been argued that hypnosis is characterized by a functional dissociation of conflict monitoring and cognitive control process [54]. Cognitive decline has been linked to class $\mathrm{C}$ occurrence decreases and class $\mathrm{B}$ duration increases in Parkinson Disease patients [55]. The four microstate classes are ordinarily alternating and the microstate syntax-for example, the link of microstates of various classes- is known to vary in various typical and pathological states [20], [29]. Accordingly, the exchange of the microstate classes may be identified with behavioral impacts and perception in Karawitan musicians.

\section{CONCLUSION}

In sum, the present microstate results revealed that Karawitan musician's brain while tuning into Gendhing Lancaran, traditional music of Java, was characterized by EEG microstate classes with the increased prominence of classes $\mathrm{A}, \mathrm{B}$, and $\mathrm{D}$ and decreased prominence of classes $\mathrm{C}$. The current investigation showed the distinctive brain activities while musicians tuned into music with respect to their musical experience utilizing EEG microstate classes. Our finding is the first study to identify the typical microstate EEG characteristics of the Karawitan musician's brains while tuning into the traditional Javanese music, Gendhing Lancaran, by using the microstate segmentaion method.

\section{ACKNOWLEDGEMENTS}

This study was partially supported by research funding from Mae Fah Luang University grant (MFU-grant no. 61210320031), Mae Fah Luang University grant (Electroencephalogram Laboratory 2019), and Graduate School of the Indonesian Institute of the Arts, Yogyakarta, Indonesia. We thank all Karawitan musicians participated as subjected in this study. The authors gratefully acknowledge the Indonesian Institute of the Arts, Yogyakarta, Indonesia, and School of Anti-Aging and Regenerative Medicine, Mae Fah Luang University, Thailand.

\section{CONTRIBUTORS}

Conceptualization: I.K. Wardani, Djohan, and P. Sittiprapaporn; Methodology: I.K. Wardani and P. Sittiprapaporn; Formal analysis: P. Sittiprapaporn; Investigation: Djohan, and P. Sittiprapaporn, F. Tyasrinestu, and P. Suyajai; Writing-original draft preparation: I.K. Wardani, and P. Sittiprapaporn; Writingreview and editing, P. Sittiprapaporn; Project administration: Djohan, and P. Sittiprapaporn. All authors have read and agreed to the published version of the manuscript. 


\section{REFERENCES}

[1] L. Patston and L.J. Tippett, "The Effect of Background Music on Cognitive Performance in Musicians and Nonmusicians," Music Perception, vol. 29, 2, pp. 173-183, 2011.

[1] G. Husain, W. F. Thompson, E. G. Schellenberg, "Effects of Musical Tempo and Mode on Arousal, Mood, and Spatial Abilities," Music Perception, vol. 20, no. 2, pp. 151-171, 2002.

[2] V. Sluming, J. Brooks, M. Howard, J. J. Downes, and N. Roberts., "Broca's Area Supports Enhanced Visuospatial Cognition in Ochestral Musicians," Journal of Neuroscience, vol. 27, no. 14, pp. 3799-3806, 2007, doi: 10.1523/JNEUROSCI.0147-07.2007.

[3] C. Gaser and G. Schlaug, "Brain Structures Differ between Musicians and Non-Musicians," Journal of Neuroscience, vol. 23, no. 27, pp. 9240-9245, 2003, doi: 10.1523/JNEUROSCI.23-27-09240.2003.

[4] G. Schlaug, "The Brain of Musicians," In: I. Peretz and R. J. Zatorre, (eds.). The Cognitive Neuroscience of Music, 2003, pp. 366-381, Oxford University Press: New York.

[5] D. Lehmann, H. Ozaki, and I. Pal, "EEG alpha map series: brain micro-states by spaceoriented adaptive segmentation," Electroencephalogr Clin Neurophysiol, vol. 67, no. 3, pp. 271-288, 1987, doi: 10.1016/0013-4694(87)90025-3.

[6] J. Wackermann, D. Lehmann, I. Dvorak, and C. M. Michel, "Global dimensional complexity of multi-channel EEG indicates change of human brain functional state after a single dose of a nootropic drug," Electroencephalogr Clin Neurophysiol, vol. 86, no. 3, pp. 193-198, 1993, doi: 10.1016/0013-4694(93)90007-I.

[7] W. Szelenberger, J. Wackermann, M. Skalski, S. Niemcewicz, and J. Drojewski, "Analysis of complexity of EEG during sleep,” Acta Neurobiologiae Experimentalis (Wars), vol. 56, no. 1, pp. 165-169, 1996.

[8] W. Szelenberger, K. Wackermann, M. Skalski, J. Drojewski, and S. Niemcewicz, "Interhemispheric differences of sleep EEG complexity," Acta Neurobiologiae Experimentalis (Wars), vol. 56, no. 4, pp. 955-959, 1996.

[9] N. Saito et al., "Global, regional, and local measures of complexity of multichannel electroencephalography in acute, neuroleptic-naive, first-break schizophrenics," Biological Psychiatry, vol. 43, no. 11, pp. 794-802, 1998, doi: 10.1016/S0006-3223(97)00547-7.

[10] C. J. Stam, E. M. Hessels-van der Leji, J. Meulstee, and J. H. R. Vilegen, "Changes in functional coupling between neural networks in the brain during maturation revealed by omega complexity," Clin Electroencephalogr, vol. 31, no. 2, pp. 104-108, 2000, doi: 10.1177/155005940003100209.

[11] D. Lehmann, W. K. Strik, B. Hemggeler, T. Koenig, and M. Koukkou, "Brain electric microstates and momentary conscious mind states as building blocks of spontaneous thinking: I. Visual imagery and abstract thoughts," International Journal of Psychophysiology, vol. 29, no. 1, pp. 1-11, 1998, doi: 10.1016/S0167-8760(97)00098-6.

[12] D. Lehmann, "Brain electric microstates and cognition: the atoms of thought", Machinery of the Mind, pp. 209-224, 1990, doi: 10.1007/978-1-4757-1083-0_10.

[13] D. Lehmann, "Consciousness: Microstates of the brain's electric field as atoms of thought and emotion," The Unity of Mind, Brain and World: Current Perspectives on a Science of Consciousness, Cambridge: Cambridge University Press; 2013. p. 191-218, 2013.

[14] T. Koenig et al., "Millisecond by millisecond, year by year: normative EEG microstates and developmental stages," Neuroimage, vol. 16, no. 1, pp. 41-48, 2002, doi: 10.1006/nimg.2002.1070.

[15] C.M. Michel and T. Koenig, "EEG microstates as a tool for studying the temporal dynamics of whole-brain neuronal networks: a review," Neuroimage, vol. 180, pp. 577-593, 2018, doi: 10.1016/j.neuroimage.2017.11.062.

[16] C.M. Michel and M.M. Murray, "Towards the utilization of EEG as a brain imaging tool," Neuroimage, vol. 61, no. 2, pp. 371-385, 2012, doi: 10.1016/j.neuroimage.2011.12.039.

[17] V. Brodbeck et al., "EEG microstates of wakefulness and NREM sleep," Neuroimage, vol. 62, no. 3, pp. 21292139, 2012, doi: j.neuroimage.2012.05.060.

[18] P. Milz, "Keypy-An open source library for EEG microstate analysis," Eur Psychiatry, vol. 33, no. S614, 2016.

[19] F. Schlegel, D. Lehmann, P. L. Faber, P. Milz and L. R. R. Gianotti, "EEG microstates during resting represent personality differences," Brain Topography, vol. 25, no. 1, pp. 20-26, 2012, doi: 10.1007/s10548-011-0189-7.

[20] L. Bréchet, D. Brunet, G. Brunet, G. Birot, R. Gruetter, C. M. Micheal, and J. Jorge, "Capturing the spatiotemporal dynamics of self-generated, task-initiated thoughts with EEG and fMRI," Neuroimage, vol. 194, pp.82-92, 2019, doi: 10.1016/j.neuroimage.2019.03.029.

[21] P. L. Faber, D. Lehmann, H. Barendregt, M. Kaelin, L. R. R. Gianotti, "Increased duration of EEG microstates during meditation," Brain Topography, vol. 18, no. 2, pp. 131, 2005

[22] A. Zanesco, A. Skwara, B. King, C. Powers, K. Wineberg, and C. Saron, "Brain Electric Microstates and Felt States of Awareness Are Modulated by Meditation Training," MindRxiv, 2019, doi: 10.31231/osf.io/rw6xf.

[23] H. Katayama, L. R. R. Gianotti, T. Isotani, P. L. Faber, K. Sasada, T. Kinoshita, and D. Lehman, "Classes of multichannel EEG microstates in light and deep hypnotic conditions," Brain Topography, vol. 20, no. 1, pp. 7-14, 2007.

[24] C. Andreou et al., "Resting-state connectivity in the prodromal phase of schizophrenia: insights from EEG microstates," Schizophrenia Research, vol. 152, no. 2, pp. 513-520, 2014.

[25] S. Irisawa et al., "Increased omega complexity and decreased microstate duration in nonmedicated schizophrenic patients," Neuropsychobiology, vol. 54, no. 2, pp. 134-139, 2006, doi: 10.1016/j.schres.2013.12.008.

[26] J. Kindler, D. Hubl, W. K. Strik, T. Dierks, and T. Koenig, "Resting-state EEG in schizophrenia: auditory verbal hallucinations are related to shortening of specific microstates," Clinical Neurophysiology, vol. 122, no. 6, pp. 1179-1182, 2011, doi: 10.1016/j.clinph.2010.10.042. 
[27] T. Koenig, D. Lehmann, M. C. G. Merlo, K. Kochi, D. Hell, and M. Koukkou, "A deviant EEG brain microstate in acute, neuroleptic-naive schizophrenics at rest," European Archives of Psychiatry and Clinical Neuroscience, vol. 249, no. 4, pp. 205-211, 1999, doi: 10.1007/s004060050088.

[28] D. Lehmann et al., "EEG microstate duration and syntax in acute, medication-naive, first-episode schizophrenia: a multi-center study," Psychiatry Research: Neuroimaging, vol. 138, no. 2, pp. 141-156, 2005, doi: 10.1016/j.pscychresns.2004.05.007.

[29] V. Strelets et al., "Chronic schizophrenics with positive symptomatology have shortened EEG microstate durations," Clinical Neurophysiology, vol. 114, no. 11, pp. 2043-2051, 2003, doi: 10.1016/S1388-2457(03)002116.

[30] A. Damborská, C. Piguetm J. M. Aubry A. G. Dayer, C. M. Michel, and C. Berchio, "Deviant EEG resting-state large-scale brain network dynamics in euthymic bipolar disorder patients," BioRxiv, 668004, 2019.

[31] K. Nishida et al., "EEG microstates associated with salience and frontoparietal networks in frontotemporal dementia, schizophrenia and Alzheimer's disease," Clinical Neurophysiology, vol. 124, no. 6, pp. 1106-1114, 2013, doi: 10.1016/j.clinph.2013.01.005.

[32] A. Tzovara, M. M. Muray, C. M. Michel, and M. De Lucia, "A tutorial review of electrical neuroimaging from group-average to single-trial event-related potentials," Developmental neuropsychology, vol. 37, no. 6, pp. 518-544, 2012, doi: 10.1080/87565641.2011.636851.

[33] D. Lehmann et al., Core networks for visual-concrete and abstract thought content: A brain electric microstate analysis," Neuroimage, vol. 49, no. 1, pp. 1073-1079, 2010, doi: 10.1016/j.neuroimage.2009.07.054.

[34] D. Lehmann et al., "EEG microstates," vol. 4, no. 3, pp. 7632, 2009.

[35] W. Strik and D. Lehmann, "Data-determined window size and space-oriented segmentation of spontaneous eeg map series," Electroencephalography and clinical neurophysiology, vol. 87, no. 4, pp. 169-174, 1993, doi: 10.1016/0013-4694(93)90016-O.

[36] J. Britz, D. C. D. Ville, and C. M. Michel, "Bold correlates of eeg topography reveal rapid resting-state network dynamics," Neuroimage, vol. 52, no. 4, pp. 1162-1170, 2010, doi: 10.1016/j.neuroimage.2010.02.052.

[37] J. Wackermann, D. Lehmann, C. M. Michel, and W. K. Strik, "Adaptive segmentation of spontaneous eeg map series into spatially defined microstates," International Journal of Psychophysiology, vol. 14, no. 3, pp. 269-283, 1993, doi: 10.1016/0167-8760(93)90041-M.

[38] D. Van de Ville, J. Britz, and C. M. Michel, "Eeg microstate sequences in healthy humans at rest reveal scale-free dynamics," Proceedings of the National Academy of Sciences, vol. 107, no. 42, pp. 18179-18184, 2010, doi: 10.1073/pnas.1007841107.

[39] P. Anderer H. V. Semlitsch, B. Saletu, and M. J. Barbanoj, "Artifact processing in topographic mapping of electroencephalographie activity in neuropsychopharmacology," Psychiatry Research: Neuroimaging, vol. 45, no. 2, pp. 79-93, 1992, doi: 10.1016/0925-4927(92)90002-L.

[40] D. Lehmann and W. Skrandies, "Reference-free identification of components of checkerboard-evoked multichannel potential fields," Electroencephalography and Clinical Neurophysiology, vol. 48, pp. 609-621, 1980, doi: 10.1016/0013-4694(80)90419-8.

[41] D. Lehmann and W. Skrandies, "Principles of spatial analysis," Elsevier: Amsterdam, In Gevins AS, Remond A (eds), Methods of Analysis of Brain Electrical and Magnetic Signals. Handbook of Electroencephalography and Clinical Neurophysiology, revised series, vol. 1, 1987, pp. 309-354.

[42] R. D. Pascual-Marqui, C. M. Michel and D. Lehmann, "Segmentation of brain electrical activity into microstates: model estimation and validation," in IEEE Transactions on Biomedical Engineering, vol. 42, no. 7, pp. 658-665, July 1995, doi: 10.1109/10.391164.

[43] B.A. Seitzman, M. Abell, S. C. Bartley, M. A. Erickson, A. R. Bolbecker, and W. P. Hetrick, "Cognitive manipulation of brain electric microstates," Neuroimage, vol. 146, pp. 533-543, 2017, doi: 10.1016/j.neuroimage.2016.10.002.

[44] T. Koenig et al., "Millisecond by millisecond, year by year: normative EEG microstates and developmental stages," Neuroimage, vol. 16, no. 1, pp. 41-48, 2002, doi: 10.1006/nimg.2002.1070.

[45] A. Custo, D. Van De Ville, W. M. Wells, M. I. Tomescu, D. Brunet, and C. M. Michel, "Electroencephalographic Resting-State Networks: Source Localization of Microstates," Brain Connect, vol. 7, no. 10, pp. 671-682, 2017, doi: 10.1089/brain.2016.0476.

[46] P. Milz, P. L. Faber, D. Lehmann, T. Koeing, K. Koci, and R. D. Pascual Marqui, "The functional significance of EEG microstates-Associations with modalities of thinking," Neuroimage, vol. 125, pp. 643-656, 2016, doi: 10.1016/j.neuroimage.2015.08.023.

[47] F. Zappasodi et al., "EEG microstates distinguish between cognitive components of fluid reasoning," Neuroimage, vol. 189, pp. 560-573, 2019, doi: 10.1016/j.neuroimage.2019.01.067.

[48] M. E. Raichle, A. M. Macleod, A. Z. Snyder, W. J. Powers, D. A. Gusnard, and G. L. Shulman, "A default mode of brain function," Proceedings of the National Academy of Sciences, vol. 98, no. 2, pp. 676-82, 2001, doi: 10.1073/pnas.98.2.676.

[49] R. D. Pascual-Marqui et al., "The resting microstate networks (RMN): cortical distributions, dynamics, and frequency specific information flow," arXiv preprint arXiv: 14111949, 2014.

[50] H. Yuan et al., "Spatiotemporal dynamics of the brain at rest--exploring EEG microstates as electrophysiological signatures of BOLD resting state networks," NeuroImage, vol. 60, no. 4, pp. 2062-72, 2012, doi: 10.1016/j.neuroimage.2012.02.031. 
[51] D. Mantini, M. G. Perrucci, C. Del Gratta, G. L. Romani, and M. Corbetta, "Electrophysiological signatures of resting state networks in the human brain," Proceedings of the National Academy of Sciences, vol. 104, no. 32, pp. 13170-13175, 2007, doi: 10.1073/pnas.0700668104.

[52] E. Pipinis et al., "Association Between Resting-State Microstates and Ratings on the Amsterdam Resting-State Questionnaire," Brain Topography, vol. 30, no. 2, pp. 245-248, 2017, doi: 10.1007/s10548-016-0522-2.

[53] H. Jiang, M. P. White, M. D. Greicius, L. C. Waelde, and D. Spiegel, "Brain activity and functional connectivity associated with hypnosis," Cerebral Cortex, vol. 27, pp. 4083-4093, 2017, doi: 10.1093/cercor/bhw220.

[54] T. Egner and J. Hirsch, "The neural correlates and functional integration of cognitive control in a Stroop task," Neuroimage, vol. 24, no. 2, pp. 539-547, 2005, doi: 10.1016/j.neuroimage.2004.09.007.

[55] C. Chu et al., "Spatiotemporal EEG microstate analysis in drug-free patients with Parkinson's disease," NeuroImage: Clinical, vol. 25, p. 102132, 2020, doi: 10.1016/j.nicl.2019.102132.

\section{BIOGRAPHIES OF AUTHORS}

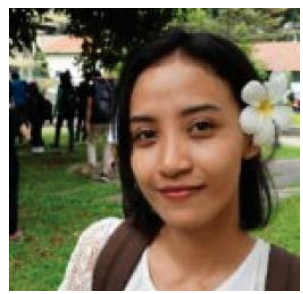

Indra K. Wardani received bachelor and master's degree of Music from Music Department, Faculty of Performing Arts, Graduate School of Indonesia Institute of the Arts Yogyakarta, Yogyakarta, Indonesia. She is currently a researcher at Music Department, Faculty of Performing Arts, Indonesian Institute of the Arts, Yogyakarta, Indonesia. Her research interest is Neuroscience of Music.

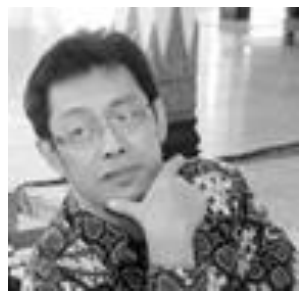

Djohan received bachelor's degree in Performing Arts from Art Institute Music School, Gadjah Mada University, Masters' degree in Psychology from Gadjah Mada University, and Doctoral degree in Psychology from Gadjah Mada University. He is currently a Professor at Music Department, Faculty of Performing Arts, Indonesian Institute of the Arts, Yogyakarta, Indonesia. His research interest is Neuroscience of Music and Arts.

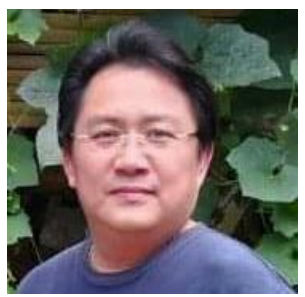

Phakkharawat Sittiprapaporn received Bachelor of Arts (Second Class Hons.) in English from Srinakharinwirot University, Thailand, Master of Arts in Linguistics, Institute of Language and Cultural for Research and Development from Mahidol University, Thailand, and Ph.D. in Neurosciences, Neuro-Behavioural Biology Center, Institute of Science and Technology for Research and Development Mahidol University, Thailand. He was the Head of Brain Science and Engineering Innovation Research Group, Mae Fah Luang University. Currently, he is the Director of the Neuropsychological Research Laboratory, as well as a lecturer at Department of Anti-Aging and Regenerative Science School of Anti-Aging and Regenerative Medicine, Mae Fah Luang University, Bangkok, Thailand. His research interests are cognitive psychology, cognitive neurosciences, cerebral mechanisms in perception and cognition, brain mechanism of music and language perception and cognition, and neurobiology of learning and memory.

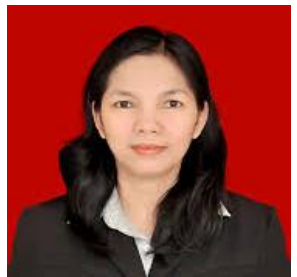

Fortunata Tyasrinestu received bachelor's degree in Music from the Indonesian Institute of the Arts, Yogyakarta and Indonesian Literature from Universitas Gadjah Mada Yogyakarta, Masters' degree in Psychology from Gadjah Mada University, and Doctoral degree in Social Sciences from Gadjah Mada University. She is Associate Professor at Music Department, Faculty of Performing Arts, Indonesian Institute of the Arts, Yogyakarta, Indonesia. She is currently a Director at Graduate School of Indonesia Institute of the Arts Yogyakarta, Yogyakarta, Indonesia. Her research interest includes Music Education and Social Sciences.

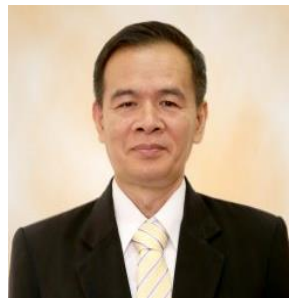

Prayoon Suyajai received bachelor's degree in Psychology from Mahachulalongkornrajavidyalaya University, Masters' degree in Clinical Psychology from Marathwda University and Educational Psychology from Srinakharinwirot University, and Doctoral degree in Psychology from Marathwda University. He is an Associate Professor and currently served as the head of Department of Psychology, Faculty of Humanities, Mahachulalongkornrajavidyalaya University, Phra Nakhon Si Ayutthaya, Thailand His research interest is Psychology. 